\title{
Harm to others reduces the sunk-cost effect
}

\author{
Zachariah I. Hamzagic $^{1}$ (D) Daniel G. Derksen ${ }^{2} \cdot$ M. Kyle Matsuba ${ }^{1} \cdot$ André Aßfalg $^{3} \cdot$ Daniel M. Bernstein $^{1,4}$
}

Accepted: 22 October 2020 / Published online: 9 November 2020

(C) The Psychonomic Society, Inc. 2020

\begin{abstract}
The sunk-cost effect (SCE) is the tendency to continue investing in something that is not working out because of previous investments that cannot be recovered. In three experiments, we examine the SCE when continued investment violates the ethic of care by harming others. In Experiment 1, the SCE was smaller if the sunk-cost decision resulted in harmful consequences towards others (an interaction between sunk cost and the ethic of care). In Experiment 2, participants considered vignettes from their own or another person's perspective. We observed an interpersonal SCE - people showed the SCE when taking the perspective of others. We did not replicate the interaction found in Experiment 1. In Experiment 3, we used statistically more powerful analyses - Bayesian sequential hypothesis testing - to examine the interaction between sunk cost and the ethic of care. We found evidence in favor of the interaction; the SCE was smaller if the sunk-cost decision harmed others. We suggest that violating one's ethic of care de-biases decision-making by overshadowing sunk costs. These findings may help explain decision-making in real-world situations involving large investments.
\end{abstract}

Keywords Decision-making $\cdot$ Sunk-cost effect $\cdot$ Moral

\section{Introduction}

In 2019, controversy emerged after two Boeing 737 Max aircrafts crashed, claiming the lives of 346 passengers (Stewart, 2019). Information surfaced that years prior, Boeing had decided to continue funding their project of installing new engines in old planes despite pilot concerns over the safety of these refurbished aircrafts (Yglesias, 2019). By the time safety concerns of the planes arose, Boeing had already committed to the project by investing a lot of time and money. Perhaps Boeing felt that they had invested too much to change course (Teger, 1980).

The "sunk-cost effect" (SCE) is the tendency to continue pursuing a venture due to previous unrecoverable investments,

Daniel M. Bernstein

Daniel.Bernstein@kpu.ca

1 Department of Psychology, Kwantlen Polytechnic University, Surrey, British Columbia, Canada

2 Department of Psychology, Simon Fraser University, Burnaby, British Columbia, Canada

3 Department of Psychology, University of Freiburg, Freiburg, Germany

4 Department of Psychology, Kwantlen Polytechnic University, 12666 72 Ave, Surrey, BC V3W 2M8, Canada despite an uncertain or unfavorable outcome (Arkes \& Blumer, 1985). The SCE has been shown in studies that require participants to imagine themselves in hypothetical vignettes (e.g., Bruine de Bruin, Strough, \& Parker, 2014), and studies where participants incur real costs rather than hypothetical ones (e.g., Hackinger, 2019). Several theories have attempted to explain the SCE, including waste avoidance theory, self-justification theory and prospect theory (Arkes, 1996; Arkes \& Blumer, 1985; Brockner, 1992; Kahneman \& Tversky, 1979). Theoretical explanations of the SCE generally adopt an intrapersonal perspective - when decision-making is based off one's own sunk costs and how the success/failure of these investments affect oneself (Olivola, 2018).

Waste-avoidance theory suggests that people are motivated not to be perceived as wasteful; thus, people generalize rules to avoid wasting unrecoverable resources (Arkes, 1996; Arkes $\&$ Blumer, 1985). Avoiding waste is useful when correctly applied, but overgeneralization of the waste-avoidance rule leads to sunk-cost decision-making if individuals refuse to terminate investments despite uncertain or negative outcomes (Larrick, Morgan, \& Nisbett, 1990).

Self-justification theory suggests that individuals avoid admitting that their previous decisions were flawed. Instead, individuals attempt to justify their previous decisions by continuing the failing cause. Additionally, a high degree of personal responsibility combined with a negative interpretation 
of the current outcome of investments increases the need for self-justification (Brockner, 1992).

Finally, prospect theory (as it pertains to the SCE; see Whyte, 1986) suggests that individuals evaluate decision outcomes based on gain and loss relative to a specific reference point rather than total gains or losses. For example, imagine that an individual brings $\$ 10$ to an arcade and loses $\$ 8$ using the claw machine without winning the prize worth $\$ 10$. Individuals are most susceptible to the SCE when they adopt a reference point that reflects their initial financial position - a loss of $\$ 8$ with a chance to return to their initial reference point by investing two more dollars and winning a prize worth $\$ 10$. Decreased outcome probability will influence decisionmaking most when the negative outcome is perceived to be certain rather than merely possible. This is because certain loss is more salient than possible loss. For example, there is no chance of winning a prize if the individual does not invest the last $\$ 2$, and there is a certain loss of $\$ 8$. However, if the individual invests the last $\$ 2$ into trying again, there is still a chance to have the investment pay off. When costs have been sunk, individuals experience a sensation of loss from the initial reference point. Therefore, one is faced with the option of accepting this certain loss or risking increased loss with further investment in hopes that the current negative outcome returns towards the initial reference point. Individuals are generally risk seeking when facing loss. Although helpful in explaining a lot of variance in sunk-cost decisions, waste avoidance theory, self-justification theory, and prospect theory, do not make explicit predictions about how harming others might moderate the SCE .

Past research has largely focused on one's own involvement in sunk-cost vignettes (see Olivola, 2018). For example, participants imagine paying for tennis lessons with their own money only to suffer an injury that makes it painful to continue with the lessons (Frisch, 1993). However, Olivola (2018) examined interpersonal dynamics by having participants imagine that investments were incurred by either themselves or others, and found that people still show the SCE in vignettes where others have incurred the sunk cost. The interpersonal vignettes used by Olivola contained no identifiable repercussions for the others in the vignettes if the sunk costs were or were not honored. This led us to question whether the SCE would be reduced in interpersonal sunk-cost vignettes where sunk-cost decisions would result in harmful effects towards others. To our knowledge no study has examined the SCE, where honoring sunk costs explicitly harms others. By considering a moral factor concerning the wellbeing of others in sunk-cost vignettes, people have the choice between two opposing decisions - continuing to honor sunk costs or looking out for the wellbeing of others.

A common understanding of morality is determining the "right" thing to do in a given situation. Moral principles often guide our behavior. According to one theoretical approach,
Moral Foundations Theory, humans possess certain innate psychological foundations of morality (Haidt \& Joseph, 2004). As a result of these innate foundations, most cultures have incorporated the importance of morality, which promotes justice, civil rights, and social welfare. Therefore, these foundations discourage harming and treating others unfairly (Graham, Nosek, Haidt, Iyer, Koleva, \& Ditto, 2011). One of the core foundations of morality is the harm/care principle, which involves compassion and concern for the wellbeing of others (Haidt, Graham, \& Joseph, 2009). Cultures then build upon these foundations to create moral systems that work to suppress selfishness and promote pro-social behavior (Haidt, 2008; Haidt, Graham, \& Joseph, 2009). Haidt et al. (2009) suggest that everyone possesses these moral foundations (e.g., the harm/care principle), but people vary in the degree to which they endorse these principles.

There is a limited literature on decision-making in sunk-cost vignettes containing moral issues. A few studies have investigated unethical business decisions using escalation of commitment - when undesirable behaviors result from multiple successive investments into a failing course of action. These studies suggest that higher magnitude of escalation (i.e., progressively investing more time/money/effort) relates to greater likelihood of unethical business decisions (Armstrong, Williams, \& Barrett, 2004; Jensen, Conlon, Humphrey, \& Moon, 2011; Street \& Street, 2006). It could be argued that unethical decisions in these studies constitute violating the harm/care principle. For example, failure to report environmental safety violations (Armstrong et al., 2004), concealing information that undermines the success of the business venture (Jensen et al., 2011) and insider trading (Street \& Street, 2006) all have the potential to harm others. However, these studies did not manipulate the presence or absence of a moral transgression in escalation of commitment vignettes. Therefore, these studies cannot examine whether committing a moral transgression reduces the likelihood of making future investments. Moreover, the effect of harm towards others in these studies was arguably implicit, because no information was provided on how decisions would directly affect others.

To our knowledge, no study has examined the SCE when honoring sunk costs explicitly violates the harm/care moral principle in vignettes where the harm clearly impacts others. We conceptualize violation of the harm/care principle as continuing with plans in the vignette despite harm to others. In three experiments, we examined how the SCE operates in vignettes containing both sunk-cost and harm/care moral principle components. We also sought to replicate the interpersonal SCE - whether people show the SCE when taking the perspective of others in sunk-cost vignettes (Olivola, 2018). We hypothesized that the harm/care principle would reduce the SCE. The harm/care principle provides a reason to end the failing cause despite previous investment. Therefore, harming another person should make people less likely to continue 
with the vignette. Further, if the harm/care component of the decision completely overshadows the sunk-cost component, harming others may eliminate the SCE entirely. In Experiment 2, we also hypothesized that individuals would show the SCE whether taking their own or another's perspective. We preregistered all experiments on the Open Science Framework (OSF) prior to any data collection. The pre-registration, including data and measures, can be found at the following link: https://osf. io/peuxr/?view_only=c273e7757a8d453195de1814114829f3

\section{Experiment 1}

\section{Method}

\section{Hypotheses}

In Experiment 1, we examined the effect of the harm/care principle on the SCE using a 2 (sunk cost: high vs. low) $\times 2$ (vignette type: harm/care vs. non-harm/care) within-subjects experimental design. The dependent variable was the likelihood rating $(0-100)$ of continuing with the vignette. We operationally defined the SCE as a positive difference score between the high sunk-cost condition and the low sunk-cost condition (high sunk cost - low sunk cost $>0$ ). We had three hypotheses:

H1: Main effect of sunk cost: Participants are more likely to indicate continuation of the vignette in high sunk-cost vignettes than low sunk-cost vignettes.

H2: Main effect of vignette type: Participants are more likely to indicate continuation of the vignette in nonharm/care vignettes than harm/care vignettes.

H3: Sunk cost by vignette type interaction: The SCE is larger in non-harm/care vignettes than harm/care vignettes.

We created and pilot-tested vignettes specifically to test these hypotheses (see Measures section).

\section{Participants}

A power analysis using $\mathrm{G}^{*}$ Power 3.1.9.2 (Faul, Erdfelder, Lang, \& Buchner, 2007) indicated a required sample size of 128 participants to detect small-to-medium effects $(\beta=.80 ; \alpha$ $\left.=.05 ; f=.15, \rho_{\mathrm{rm}}=.5\right) .{ }^{1} \mathrm{~A}$ meta-analysis found that the SCE averages a medium effect size, but is moderated by many

\footnotetext{
${ }^{1}$ For anyone not familiar with power calculations, the user interface of GPower is not transparent about power calculations for interaction effects. In Experiments 1 and 2, we settled for the ability to detect a $2 \times 2$ interaction with numerator $\mathrm{df}=1$ and denominator $\mathrm{df}=N-4$. In G-Power, this was achieved by selecting "ANOVA: Repeated measures, within factors," "Number of groups = 4 ," and "Number of measurements $=2$."
}

different factors (Roth, Robbert, \& Straus, 2015). Despite this, we decided to power for a small-to-medium effect size for two reasons: (1) We included both previously adapted and newly created materials in our design; and (2) we examined the SCE in a harm/care moral context, which has not been done previously. We recruited participants via Amazon's Mechanical Turk (MTurk). Participants received US\$4 for their participation. To ensure a reliable sample and to avoid random responding from bots, we only recruited MTurkers who had completed a minimum of 5,000 MTurk tasks with a cumulative approval rating of $99 \%$. We planned to exclude participants who answered more than one attention-check question incorrectly. Participants followed a link to the online survey medium, Qualtrics, where they completed the remainder of the study. Of a total sample of 158 participants, we excluded data from 28 participants who withdrew from the study during data collection. No data were excluded because of random responding on attention-check questions.

Of the remaining 130 participants, $82(63.1 \%)$ were male, $47(36.2 \%)$ were female and one participant identified as nonbinary. The ages of the participants ranged from 20 to 72 years, with an average age of 35.5 years. The majority of the participants self-reported as Caucasian $(75.4 \%)$, followed by Asian (8.5\%), African-American (7.7\%), and other (8.5\%).

\section{Measures}

Sunk-cost vignettes We created 12 sunk-cost vignettes for this study (see Appendix). We adapted nine vignettes from previous sunk-cost literature (Bruine de Bruin, Strough, \& Parker, 2014; Frisch, 1993; Morsanyi \& Handley, 2008; Olivola, 2018; Strough, Schlosnagle, Karns, Lemaster, \& Pichayayothin, 2014), and created three specifically for this study. Adapted vignettes followed similar narratives as those used in previous sunk-cost research, except for the addition of a harm/care component. Each vignette differed by the amount of sunk cost and vignette type, resulting in four conditions per vignette: (1) low sunk cost, non-harm/care; (2) low sunk cost, harm/care; (3) high sunk cost, non-harm/care and; (4) high sunk cost, harm/care. An example of a high sunk cost harm/care vignette was, "Imagine that you are babysitting your niece and nephew, and you decide to watch a movie with them. You take them to the theater and spend $\$ 50$ on movie tickets. You are excited to see this movie based on the trailer. After $5 \mathrm{~min}$, one of the kids finds the movie scary, and is terrified to watch it. How likely are you to continue watching the movie?" In the low sunk-cost conditions, the movie tickets are free vouchers, and in the non-harm/care conditions, the movie is boring. We pilot tested all vignettes prior to data collection and examined the means for the sunk-cost and vignette type conditions to ensure the vignettes were being treated as we intended (i.e., more likely to continue with the vignette in the high sunk-cost versus the low sunk-cost condition, and the non-harm/care versus the harm/ 
care condition). For all vignettes, participants used a slider scale of $0-100$ to indicate the likelihood of continuing with the vignette.

\section{Procedure}

Participants answered a sunk-cost question for 12 different vignettes in counterbalanced order, following a Latin-square design by completing only one of the four conditions for each vignette. Following completion of the sunk-cost vignettes, participants completed a battery of personality measures for exploratory purposes, which we do not discuss further. To detect random responding, we embedded five attentioncheck questions throughout the study. These questions asked participants not to respond and to proceed to the next question. Upon completing the study, participants were thanked and debriefed.

\section{Results}

In Experiment 1, we examined whether the harm/care principle influenced the SCE using a 2 (sunk cost: high vs. low) $\times 2$ (vignette type: harm/care vs. non-harm/care) repeatedmeasures ANOVA, with the dependent variable being the likelihood to continue with the failing cause $(\alpha=.05)$.

The results of Experiment 1 appear in Fig. 1. Confirming $H 1$, participants were more likely to continue with the vignette in the high sunk-cost condition compared to the low sunk-cost condition, $F(1,126)=9.21, p=.003, \eta_{\mathrm{p}}{ }^{2}=.07$. Confirming $H 2$, participants were also more likely to continue with the vignette in the non-harm/care condition than the harm/care condition, $F(1,126)=97.2, p \leq .001, \eta_{\mathrm{p}}{ }^{2}=.44$. Confirming $H 3$, the sunk cost $\times$ vignette type interaction was also significant, $F(1,126)=4.41, p=.04, \eta_{\mathrm{p}}{ }^{2}=.03$. The SCE was significant in the non-harm/care condition, $F(1,126)=18.33$,

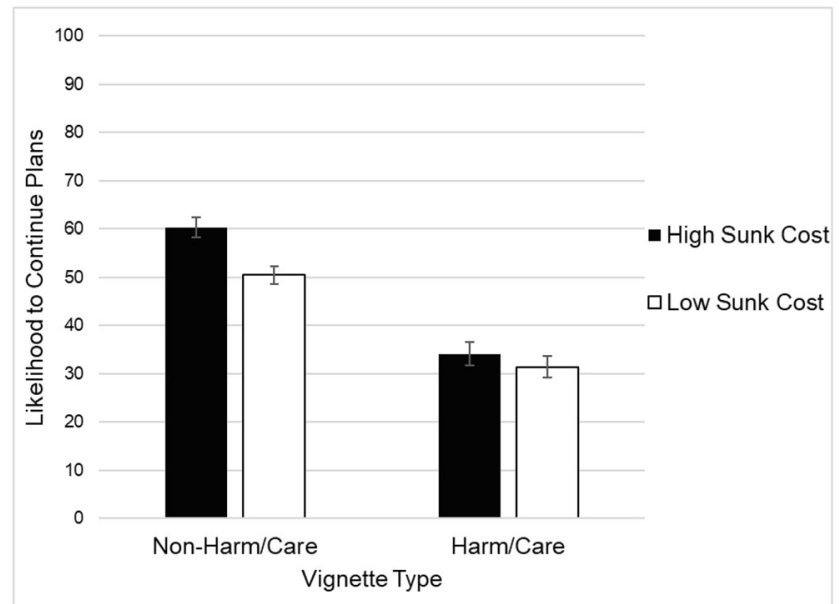

Fig. 1 Experiment 1 likelihood to continue with plans across sunk cost and vignette type. Error bars represent standard error of the mean $p \leq .001, \eta_{\mathrm{p}}{ }^{2}=.13$, but not in the harm/care condition, $F(1,126)=.80, \eta_{\mathrm{p}}^{2}=.006$.

\section{Discussion}

Experiment 1 examined how violating the harm/care principle influences the SCE. Consistent with previous research, we found evidence for the SCE (Arkes \& Blumer, 1985). The results also suggest that people are less likely to continue with a sunk-cost vignette when it would harm others. We argue that the moral transgression of harming others influenced decision-making more than the sunk-cost aspect, in some cases enough to eliminate the SCE entirely.

\section{Experiment 2}

Experiment 2 sought to replicate the results observed in Experiment 1, and show the interpersonal SCE found by Olivola (2018). We did not use the same materials or design as Olivola; thus, Experiment 2 served as a conceptual replication of the interpersonal SCE. We had four hypotheses:

H1: Main effect of sunk cost: Participants are more likely to indicate continuation of the vignette in high sunk-cost vignettes than low sunk-cost vignettes.

H2: Main effect of vignette type: Participants are more likely to indicate continuation of the vignette in nonharm/care vignettes than harm/care vignettes.

H3: Sunk cost by vignette type interaction: The SCE is larger in non-harm/care vignettes than harm/care vignettes.

H4: No effect of person in vignette: There is no difference in decision-making whether taking one's own perspective, or the perspective of another person in the vignettes.

\section{Method}

\section{Participants}

Experiment 2 was a 2 (sunk cost: high vs. low) $\times 2$ (vignette type: harm/care vs. non-harm/care) $\times 2$ (person in vignette: self vs. other) mixed design with person in vignette as the only between-subjects variable. We used the power analysis from Experiment 1 to determine our sample size for Experiment 2. From the total sample of 161 participants, we excluded data from 31 participants who withdrew from the study during data collection. No data were excluded because of random responding on attention-check questions.

Of the remaining 130 participants, $83(63.8 \%)$ were male, and $47(36.2 \%)$ female. The age of the participants ranged from 19 to 84 years, with an average of 33.5 years. The 
majority of the participants self-reported as Caucasian $(70.8 \%)$, followed by Asian (10\%), African-American $(9.2 \%)$, Hispanic $(5.4 \%)$, and other $(4.6 \%)$. The data collection procedure, including compensation for participation, was the same as Experiment 1.

\section{Measures}

All measures were identical to Experiment 1, except for the addition of the perspective of the person in the vignettes (self/other). Therefore, each vignette had a self-version (e.g. "Imagine that you...") and an other-version (e.g. "Imagine that Bradley..."). The others in the vignettes were hypothetical people, and we did not indicate that they have any relation to the participant.

\section{Results}

We conducted a 2 (sunk cost: high vs. low $) \times 2$ (vignette type: harm/care vs. non-harm/care) $\times 2$ (person in vignette: self vs. other) ANOVA with person in vignette as the only betweensubjects variable. The mean $(S E)$ likelihood judgments for each condition appear in Fig. 2. Confirming H1, we found that participants were more likely to continue with the vignette in the high sunk-cost condition than the low sunk-cost condition, $F(1,128)=17.42, p \leq .001, \eta_{\mathrm{p}}{ }^{2}=.12$. Confirming $H 2$, we also found that participants were more likely to continue with the vignette in the non-harm/care condition than in the harm/ care condition, $F(1,128)=171.11, p \leq .001, \eta_{\mathrm{p}}{ }^{2}=.57$. Disconfirming $H 3$, the sunk-cost $\times$ vignette type interaction was not significant $F(1,128)=1.06, p=.31, \eta_{\mathrm{p}}{ }^{2}=.01$. Confirming $H 4$, there was no effect of person incurring the cost on the $\operatorname{SCE} F(1,128)=1.11, p=.29, \eta_{\mathrm{p}}{ }^{2}=.01$.

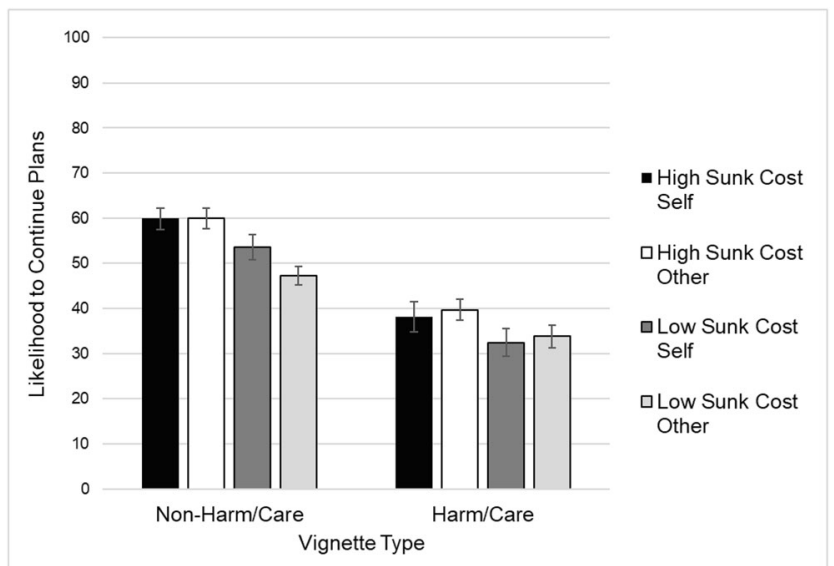

Fig. 2 Experiment 2 likelihood to continue with plans across sunk cost and vignette type. Error bars represent standard error of the mean

\section{Discussion}

In Experiment 2 we attempted to replicate Experiment 1 and find evidence for the interpersonal SCE. Participants showed the SCE and were less likely to continue with a vignette when doing so would cause harm to others regardless of the size of the investment. We further found an interpersonal SCE, whereby participants were equally susceptible to the SCE, regardless of a self or other perspective (Olivola, 2018). However, contrary to Experiment 1, there was no interaction between sunk cost and vignette type. There are several potential reasons for this discrepancy. Because we introduced the self/other between-subjects variable in Experiment 2, it was not a direct replication of Experiment 1, and may have influenced our ability to observe the interaction between sunk cost and vignette type. We also used nondirectional tests in the form of ANOVA to test a directional hypothesis. Specifically, we hypothesized that harming others should reduce the SCE. We addressed these concerns in Experiment 3.

\section{Experiment 3}

Experiment 3 was a direct replication of Experiment 1. Our main interest in Experiment 3 was to examine the interaction observed in Experiment 1 which was not replicated in Experiment 2. We introduced several improvements to the statistical analysis to test for the interaction in Experiment 3 by using Bayesian analyses which we did not use in Experiments 1 and 2.

\section{Method}

\section{Hypotheses and analysis procedures}

In Experiment 2, we did not observe the interaction effect between sunk cost and vignette type that we observed in Experiment 1 . This is a common dilemma in statistical analysis, as there could be two possibilities: Either the interaction does not exist, or it does exist and our statistical analysis was underpowered. Although the power analysis we conducted to plan the sample sizes in Experiments 1 and 2 should overcome this issue, it suffers from a fundamental weakness: Unless the expected effect size is correctly anticipated, the statistical test can be underpowered. A solution to this problem is sequential hypothesis testing (e.g., Schönbrodt, Wagenmakers, Zeheleitner, \& Perugini, 2017). A sequential test includes the comparison of two mutually exclusive hypotheses. If the evidence for either hypothesis is only moderate, the data collection continues. The data collection ends when the evidence is extreme enough in favor of one of the hypotheses. This approach elegantly solves the power issue as part of the data collection: while the study is underpowered, it is extremely 
unlikely to observe enough evidence for either hypothesis and, consequently, to stop the data collection.

We implemented a sequential testing strategy in Experiment 3 with Bayesian $t$-tests (Rouder, Speckman, Sun, Morey, \& Iverson, 2009). Conventional significance tests provide a $p$-value indicating statistical significance, but Bayesian hypothesis tests offer a numerical value called the Bayes Factor (BF). In our analyses, the BF expresses evidence in favor of an interaction between sunk cost and vignette type relative to the evidence against this interaction. A BF of 10, for example, would suggest odds of 10 for the presence of an interaction effect, whereas a BF of $1 / 10$ would suggest odds of 10 for the absence of an interaction effect. Thus, compared to the conventional significance test, the BF can express evidence in favor of a null hypothesis instead of being inconclusive when $p>.05$. We assessed the BF every time a new group of 20-25 participants had contributed to the data set and determined the end of the data collection based on the available evidence provided by the $\mathrm{BF}$. This sequential testing strategy requires fewer participants than comparable fixed-sample size tests such as the ANOVA we used in Experiments 1 and 2 (e.g., Wald, 1945). We further opted for Bayesian $t$-tests in Experiment 3 rather than ANOVA because we tested directional hypotheses consistent with the observed interaction in Experiment 1. Directional hypotheses improve the statistical power compared to exploratory analyses such as the ANOVA in Experiments 1 and 2.

We predicted that the SCE is lower in the harm/care condition than the non-harm/care condition $(H 1)$. A prerequisite for $\mathrm{H} 1$ is the presence of the SCE in the non-harm/care condition (H2). For each participant we computed the difference score of the participants' mean likelihood rating of continuing with the vignette in the high sunk-cost condition minus the mean likelihood rating in the low sunk-cost condition.

Regarding hypothesis $H 1$ we compared the statistical hypotheses:

H1.0: SCE in harm/care vignettes $\geq \mathrm{SCE}$ in non-harm/ care vignettes

H1.1: SCE in harm/care vignettes < SCE in non-harm/ care vignettes via the Bayes Factor (BF) in a pairedsamples Bayesian $t$-test. Regarding hypothesis $\mathrm{H} 2$ we compared the statistical hypotheses:

H2.0: SCE in non-harm/care vignettes $\leq 0$

$\mathrm{H} 2.1$ : SCE in non-harm/care vignettes $>0$

via the $\mathrm{BF}$ in a one-sample Bayesian $t$-test. As stoppage criterion for data collection, we defined four conditions that would end the data collection: (1) if the sample size reached 300 participants; (2) if we observed a BF > 10 in favor of hypothesis $\mathrm{H} 2.0$ which would indicate that we failed to replicate the SCE in Experiment 3; (3) if we observed that H1.0 was supported by a $\mathrm{BF}>10$; and, (4) if we observed that H1.1 was supported by a $\mathrm{BF}>10$.
The mutually exclusive third and fourth conditions helped us clarify whether the SCE was lower in a harm/care context. We tested whether a stoppage criterion was met after running a new batch of participants, excluding participants that met at least one of the exclusion criteria (see the Participants section). For the Bayesian analyses, we used the $\mathrm{R}$ package BayesFactor with default priors (R Core Team, 2019).

\section{Participants}

Participant recruitment was the same as in Experiments 1 and 2 , except that participants received US\$2 for their participation. In the first two experiments we overestimated the time we thought it would take participants to finish the task (40 min). In Experiment 3, we adjusted our compensation according to the average time it actually took participants $(20 \mathrm{~min})$. Additionally, to ensure that participants were proficient in reading English we also administered the LexTALE language proficiency measure (Lemhöfer \& Broersma, 2012). In this task, participants make a lexical decision on 60 letter strings, indicating if it is a proper English word or not. We excluded participants who scored less than $80 \%$ correct identification. Of a total sample of 232 participants, we excluded data from 36 participants because of an insufficient LexTALE performance and 15 participants who withdrew from the study during data collection.

Of the remaining 181 participants, $102(56.3 \%)$ were male, $78(43.1 \%)$ female and one participant identified as genderfluid. The age of the sample ranged from 20 to 73 years, with an average age of 37 years. The majority of participants selfreported as Caucasian (79\%), followed by African-American (7.2\%), Asian (5\%), Hispanic (5\%), and other (3.9\%) participants.

\section{Measures}

The vignettes were identical to Experiment 1, with one exception. To investigate the interaction between the sunk-cost and harm/care conditions, we needed to ensure that we observed the strongest possible SCE in the non-harm/care condition with our materials. To that end, we removed the four vignettes that proved to be the least effective in producing the SCE in Experiments 1 and 2. We included the remaining eight vignettes in Experiment 3 (see Appendix).

\section{Results}

We reached one of the pre-registered stoppage criteria with a sample of 128 participants (after exclusions): The BF for hypothesis H1.1 was greater than 10. Thus, the SCE was lower in the harm/care condition compared to the non-harm/care condition. However, we decided to collect more data because we were at this point well below our pre-registered maximum 
sample size of 300 participants and we wanted to strengthen the evidence for or against an interaction of sunk cost (high vs. low) and vignette type (harm/care vs. non-harm/care). We ended the data collection with a sample of 181 participants (after exclusions). The evidence had further increased for the interaction tested in hypothesis H1.1 without any qualitative changes to the results compared to the sample of 128 participants. We provide the data and $\mathrm{R}$ analysis file of Experiment 3 on the OSF platform.

The mean likelihood ratings to continue with plans in Experiment 3 appear in Fig. 3. Like Experiments 1 and 2, we found a SCE in the non-harm/care vignette condition (hypothesis H2.1: SCE in non-harm/care vignettes $>0$ ): the difference score between the mean likelihood rating of continuing with the vignette in high sunk-cost condition minus low sunk-cost condition (i.e., SCE) was greater than zero $(M=$ 9.83, $S D=28.63), \mathrm{BF}=2.31 \mathrm{e} 5$. In comparison, the $\mathrm{SCE}$ was lower in the harm/care vignette condition $(M=4.29, S D$ = 26.09), supporting hypothesis H1.1 (SCE in harm/care vignettes $<\mathrm{SCE}$ in non-harm/care vignettes), $\mathrm{BF}=27.90$. Observing the support for hypothesis H1.1, we were curious whether the SCE was present in the harm/care condition. We, therefore, tested the hypotheses H3.0, SCE in harm/care vignettes $\leq 0$ and H3.1, SCE in harm/care vignettes $>0$ against each other (we did not pre-register this test). We found strong support for $\mathrm{H} 3.1$ compared to $\mathrm{H} 3.0, \mathrm{BF}=66.71$, suggesting that the SCE was still present in the harm/care condition. Thus, in Experiment 3, the SCE appeared regardless of vignette type but was lower in the context of harm/care vignettes compared to non-harm/care vignettes.

Unfortunately, there is no significance test that would be able to test hypothesis H1.0 (SCE in harm/care vignettes $\geq$ $\mathrm{SCE}$ in non-harm/care vignettes) against hypothesis H1.1 (SCE in harm/care vignettes < SCE in non-harm/care vignettes). However, to satisfy a reviewer request, we ran a 2 (sunk cost: high vs. low) $\times 2$ (vignette type: harm/care vs. nonharm/care) repeated-measures ANOVA, with likelihood to continue with the failing cause as dependent variable $(\alpha=$ .05 ). Participants were more likely to continue with the vignette in the high sunk-cost condition compared to the low sunk-cost condition, $F(1,180)=26.41, p \leq .001, \eta_{\mathrm{p}}{ }^{2}=.13$. Participants were also more likely to continue with the vignette in the non-harm/care condition than the harm/care condition, $F(1,180)=265.75, p \leq .001, \eta_{\mathrm{p}}{ }^{2}=.60$. The sunk cost $\times$ harm/care interaction did not reach significance, $F(1,180)=$ $3.40, p=.067, \eta_{\mathrm{p}}{ }^{2}=.02$. However, a statistically more powerful one-sided $t$-test with SCE as dependent variable suggested that the SCE was greater in the non-harm/care than the harm/care condition, $t(180)=1.84, p=.03, d=.137$. Please note that the ANOVA interaction and the $t$-test both refer to the null hypothesis: $\mathrm{SCE}$ in harm/care vignettes $=\mathrm{SCE}$ in non-harm/care vignettes. This is only a subset of hypothesis H1.0 and therefore insufficient for present purposes.
Significance tests further only express evidence against a null hypothesis instead of testing hypotheses such as H1.0 and H1.1 against each other. Although the significance tests fail to address the focal research question of Experiment 3, they better mirror the analysis of Experiments 1 and 2.

\section{Discussion}

In Experiment 3 we attempted to replicate Experiment 1 and find evidence for or against the interaction effect between the sunk-cost and harm/care conditions using Bayesian analyses. Replicating both Experiment 1 and Experiment 2, participants showed the SCE and were less likely to continue with the vignette when it would harm others. Most critically, we found evidence for the interaction effect reported in Experiment 1: Harming others reduced the SCE.

\section{General discussion}

We examined the effects of the harm/care moral principle on the SCE. In three experiments, we found evidence for the SCE: Participants were more likely to continue with the vignette when an initial investment was high compared to low. We also found that participants were generally more inclined to continue with the vignette in non-harm/care vignettes compared to harm/care vignettes. This was the case irrespective of sunk costs and irrespective of whether a self-or-other perspective was used (Experiment 2). More importantly, we wondered whether the SCE itself would be smaller when continuing with the vignette violated the harm/care principle. After initial evidence for this hypothesis in Experiment 1, we did not find support for this hypothesis in Experiment 2. We resolved this in Experiment 3 by employing a statistically more powerful analysis consistent with our directional hypothesis. Specifically, we used a Bayesian sequential $t$-test in

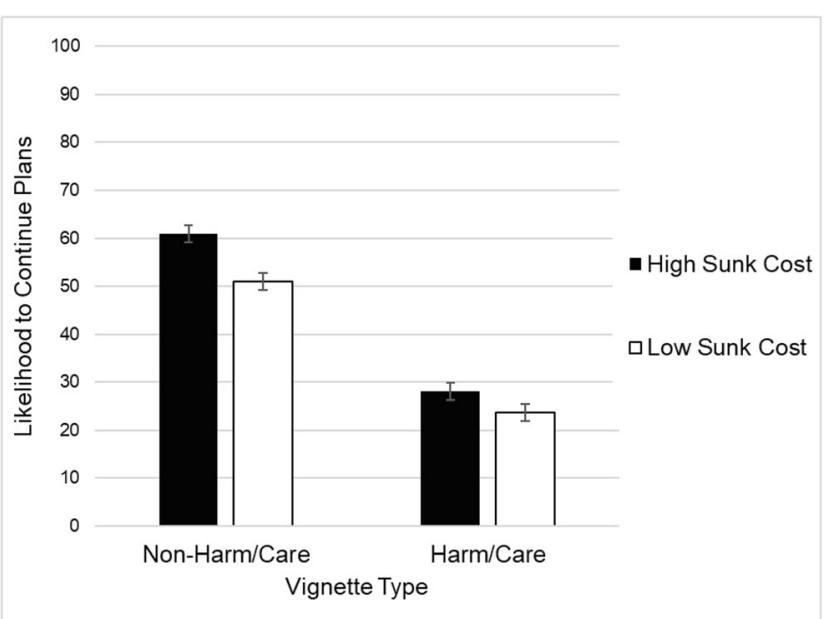

Fig. 3 Experiment 3 likelihood to continue with plans across sunk cost and vignette type. Error bars represent standard error of the mean 
Experiment 3 to decide when to stop the data collection. With this improved procedure, we found support for the interaction between sunk cost and vignette type that we observed in Experiment 1: The SCE was smaller in harm/care vignettes where the continuation of a vignette harmed others, thereby violating the harm/care moral principle. In Experiment 2, we also found evidence for the interpersonal SCE. Participants showed the SCE when taking the perspective of another person in the vignette, conceptually replicating Olivola (2018).

Our results may identify a factor that de-biases sunk-cost decision-making: one's care towards others. Although the SCE is a robust effect that occurs in many areas like committed relationships, financial investments, and public support for war, other aspects relevant to a particular decision may be salient enough to influence decision-making above sunk costs (i.e., the harm/care principle; Guler, 2007; Rego, Arrantes \& Magalhães, 2018; Schott, Scherer, \& Lambert, 2011). In our vignettes, the sunk-cost and ethic of care aspects influence opposite decisions. Sunk costs influence continuation of the vignette, whereas the ethic of care influences discontinuation. Note that our results are limited to the specific way we constructed the vignettes - when honoring sunk costs harms others. We would expect to find the opposite effect in vignettes where failing to honor sunk costs harms others. That is, we would expect a larger SCE in these cases.

More salient aspects of the vignette may have a stronger influence on decision-making. Levin, Schneider, and Gaeth (1998) suggest that avoiding negative consequences of a behavior (i.e., not harming others) is a more salient goal than attaining positive consequences (i.e., having prior investments pay off). It may also be that consequences of more salient information (i.e., harming others) are more easily imagined than consequences of less salient information (i.e., poor investment), which is more influential in guiding judgement and decision-making (Sherman, Cialdini, Schwartzman, \& Reynolds, 1985). The current study found that violating the harm/care moral principle (i.e., harming others) reduced the SCE - there was a smaller SCE in the harm/care vignettes compared to non-harm/care vignettes. It may be that harming others is more salient than sunk cost, thereby influencing decisions to a higher degree. We suggest that violating one's ethic of care de-biases decision-making by overshadowing sunk costs.

The results of the current study also served as the first conceptual replication we are aware of regarding the interpersonal SCE found by Olivola (2018). The interpersonal SCE challenges the notion that people often incorrectly believe that others make more rational decisions than themselves (Greenstein \& Xu, 2015). It is often assumed that people make sunk-cost decisions because of how they themselves are affected by the outcome. It is possible that participants may simply run a mental simulation on how they would react, and then indicate that someone else would do the same (Shanton \&
Goldman, 2010). Overall, the current study suggests the need for future research to consider interpersonal dynamics when examining the SCE.

The present research does not challenge any theory that explains the SCE. Rather, the present work highlights one particular blind spot for several SCE theories: they have not formally addressed how moral principles, such as harm/care, may moderate sunk-cost decisions. As social beings, the impact of our choices on others is a relevant factor to consider when making-decisions. Waste-avoidance theory suggests that people are motivated not to be perceived as wasteful; thus, people generalize rules to avoid wasting unrecoverable resources (Arkes, 1996; Arkes \& Blumer, 1985). This theory overlooks the possibility of other factors (like moral transgressions) in sunk-cost situations that also motivate decision-making. Decision-makers may feel that it is more important to avoid harming others than being perceived as wasteful.

Self-justification theory states that individuals seek to avoid admitting that previous investments were a mistake by continuing a failing cause (Brockner, 1992). This theory fails to account for situations where there are salient factors other than sunk costs that may influence one's desire to see themselves as competent decision-makers. It may be more difficult for someone to justify decisions that result in harm to others than poor investments, especially individuals who view morality as an integrated part of their identity (Reynolds \& Ceranic, 2007). Therefore, knowingly harming others may itself act as an acceptable reason to escape the need to justify poor investments.

Prospect theory suggests that people make decisions regarding an outcome based on loss and gain relative to a reference point (e.g., before any investment or according to their current situation). In non-harm/care vignettes, individuals have a choice between a certain smaller loss (e.g., \$50 sunk into the boring movie) and possible larger loss (e.g., more investment of time watching the movie plus the initial \$50 if the movie doesn't improve). Recall that individuals are riskseeking when making a choice between certain smaller loss and larger potential loss. In a sunk-cost harm/care situation, individuals may perceive a choice between two certain losses - the initial investment and the consequences associated with harming others. If this assumption is correct, individuals may perceive the loss associated with harming others (and the losses of the investment of time/money) as greater than the loss of the initial investment. Therefore, individuals choose the lesser of the certain losses. For example, individuals prefer to accept a loss of $\$ 50$ than deal with the consequences of the children being scared plus the loss of the cost of the movie. However, we do not know whether harm caused to others is perceived as a loss in the same way that one perceives loss of resources like time and money.

Literature in moral philosophy has begun to explore this general topic. In moral sunk-cost scenarios, the decision to 
continue in war is often used as the example where the sunkcost is the loss of lives. The question that emerges is whether we continue to incur costs when the war is going badly. Some individuals may adopt a prospect view, whereby the moral cost of past loss of life should not factor into future decisionmaking (e.g., 10,000 dead soldiers cannot be revived and should not affect the decision of whether to continue a war). Others may adopt quota or discount views. The quota view fully accounts for each moral cost and sets a fixed limit, and once the moral cost limit is reached (e.g., 10,000 soldier deaths) we should withdraw from the war. The discount view offers a proportional value to prior moral costs (e.g., each of the 10,000 lives lost so far counts as 0.8 , allowing for 2,000 more deaths to occur before the 10,000 limit is reached; see Lazar, 2018; Uzan, 2020). These theories apply to decisionmaking in the moral domain where the moral cost is often the loss of lives. Our current work focuses on the economic cost of decision-making when moral considerations are introduced.

In our research, we do not suggest that these theories fail to explain the sunk-cost effect. Rather, it may be problematic to assume that a single theory which focuses on a specific aspect of a situation (i.e., waste, gain/loss, self-justification) can explain decision-making in all sunk-cost situations. In certain situations, there may be other salient factors that also influence decision-making. Violating the ethic of care and harming others may be one such factor.

In the current study, we did not examine the mechanism underlying how violating a moral care principle reduces the SCE. Future research should seek to answer this question. Furthermore, future studies should test whether salient factors other than the harm/care principle reduce the SCE. Future research might also manipulate the escalation of sunk costs in the presence of a harm/care transgression to examine whether there is a point at which sunk costs are more important than harming others. Recall that the possibility of harming others did not dissuade Boeing from discontinuing their investment to produce 737 aircrafts despite known safety concerns. It may be that we have the benefit of hindsight that places the blame squarely on Boeing for failing to change its course. It may, however, be that there is a point of no return, beyond which even moral transgressions do not outweigh sunk costs.

Future research should examine whether transgressing other moral principles like fairness (eliciting concerns of equality and more complex concepts of justice) or loyalty (eliciting concerns related to betrayal and in-group membership; Haidt, Graham, \& Joseph, 2009), or normative ethical principles (utilitarianism) have the same debiasing effect in sunkcost vignettes. If so, then there may be a general moral effect that is causing the debiasing. Finally, it may also be worthwhile to examine how individual differences like personality traits and political ideology interact with vignettes containing both sunk costs and moral transgressions. It is important to note that, despite our best effort to depict scenarios that harm others, it is possible that participants were motivated to discontinue investment for reasons other than concern for the wellbeing of others. Several of the vignettes depict scenarios that contain consequences for honoring sunk costs other than people being harmed. Two examples of this are angry parents of the kids taken to the scary movie, or revoked privileges for further babysitting opportunities. Therefore, there may be other factors in some vignettes that influenced decision-making that we did not anticipate. It should also be noted that because the interactions observed in Experiments 1 and 3 do not cross over, they are potentially removable (see Loftus, 1978, and Wagenmakers, Krypotos, Criss, \& Iverson, 2012). Future studies could explore ways to observe a crossover interaction (e.g., adjusting materials to remove the main effect of vignette type or eliminate the SCE entirely in harm/care vignettes). Overall, we found that harming others reduces the SCE and de-biases sunk-cost decision-making.

Acknowledgements This work was supported by grants from the Canada Research Chairs Program (950-228407), the Social Sciences and Humanities Research Council of Canada (435-2015-0721), the Kwantlen Polytechnic University Office of Research and Scholarship, and the German Research Foundation (DFG: AS 427/3). We thank Geoff Loftus for helpful comments on an earlier draft of this work. Results of the first two experiments in this study were presented as a talk at the annual North West Cognition and Memory conference in 2019.

Author note Z. I. Hamzagic developed the study concept. Z. I. Hamzagic, D. G. Derksen, K. Matsuba and D. M. Bernstein contributed to the study design. Testing and data collection were performed by Z. I. Hamzagic. Data analysis and interpretation were performed by Z. I. Hamzagic and A. Aßfalg (Experiment 3 only). Z. I. Hamzagic drafted the manuscript, and D. G. Derksen, A. Aßfalg, M. K. Matsuba and D. M. Bernstein provided critical revisions. All authors approved the final version of the manuscript for submission.

\section{Compliance with ethical standards}

Conflict of interest None.

The data and materials for all experiments are available at https://osf. io/peuxr/?view_only=c273e7757a8d453195de1814114829f3. All three Experiments were pre-registered, and can be seen using the link above.

\section{Appendix}

\section{Materials}

\section{Vignette 1: Concert}

\section{Adapted from Olivola (2018) Non-Harm/Care Conditions:} Imagine that you (spent $\$ 200$ on tickets) won free tickets for you and a friend to front row seats of a concert to see your favorite performer. However, a storm makes travelling to the concert very cold, slow and potentially hazardous. 
Harm/Care Conditions: Imagine that you (spent \$200 on tickets) won free tickets for you and a friend to front row seats of a concert to see your favorite performer. However, before you leave to the concert, your friend comes down with a migraine. The loud music will only make it worse.

Choice: How likely are you to continue with your plans to go to the concert?

\section{Vignette 2: Movie}

Adapted from Strough et al. (2014) Non-Harm/Care Conditions: Imagine that you are babysitting your niece and nephew, and you decide to watch a movie with them. You take them to the theater (and spend $\$ 50$ on movie tickets) with free movie vouchers. You are excited to see this movie based on the trailer. After $5 \mathrm{~min}$, you are very bored and the movie seems really bad.

Harm/Care Conditions: Imagine that you are babysitting your niece and nephew, and you decide to watch a movie with them. You take them to the theater (and spend \$50 on movie tickets) with free movie vouchers. You are excited to see this movie based on the trailer. After 5 min, one of the kids finds the movie scary, and is terrified to watch it.

Choice: How likely are you to continue watching the movie?

\section{Vignette 3: Bike}

Created by authors Non-Harm/Care Conditions: Imagine that your car breaks down and will be in the shop for 3 weeks to get fixed. Along with another co-worker, you decide that this is a good opportunity to start biking to work in order to live a healthier lifestyle. You get a (non-refundable bike for \$300) bike for free. After two trips to work, you realize you dislike the bike ride since it is harder and takes longer than you expected.

Harm/Care Conditions: Imagine that your car breaks down and will be in the shop for 3 weeks to get fixed. Along with another co-worker, you decide that this is a good opportunity to start biking to work in order to live a healthier lifestyle. You get a (non-refundable bike for \$300) bike for free, and after two trips to work, you find out that the bike was stolen and that the owner is looking for it.

Choice: How likely are you to keep the bike?

\section{Vignette 4: Skate}

Adapted from Morsanyi and Handley (2008) Non-Harm/ Care Conditions: Imagine that you and a friend want to skate, so you go to the ice rink. (You pay $\$ 25$ for) Admission and skate rental is free, and you have one hour to skate. When you step on to the ice, you find the rink so crowded with people that it's impossible to move around and you don't enjoy the whole thing at all.

Harm/Care Conditions: Imagine that you and a friend want to skate, so you go to the ice rink. (You pay $\$ 25$ for) Admission and skate rental is free, and you have one hour to skate. When you step on to the ice, your friend immediately falls and sprains their ankle. It hurts to continue skating.

Choice: How likely are you to continue skating for the rest of the hour?

\section{Vignette 5: Dinner *Removed from Experiment 3}

Adapted from Strough et al. (2014) Non-Harm/Care Conditions: Imagine that you spent $30 \mathrm{~min}$ (and spend $\$ 100$ on ingredients) using ingredients from home to prepare a meal for your family using a new recipe. After testing a few bites of it, you realize that you dislike the taste of it. Even after adding some spices, you still dislike the taste of it.

Harm/Care Conditions: Imagine that you spent $30 \mathrm{~min}$ (and spend \$100 on ingredients) using ingredients from home to prepare a meal for a dinner party that you are hosting. One of your guests mentions that they have celiac disease and does not eat gluten because it will cause severe discomfort. However, the ingredients you used are not gluten free.

Choice: How likely are you to serve the meal?

\section{Vignette 6: Amusement Park}

Created by authors Non-Harm/Care Conditions: Imagine that you take your niece and nephew to an amusement park for the day. You (pay $\$ 150$ for) get the three admission tickets for free. However, once you enter the park, you realize the park is very busy and the line for each ride is at least an hour long, and it is a very hot day.

Harm/Care Conditions: Imagine that you take your niece and nephew to an amusement park for the day. You (pay $\$ 150$ for) get the three admission tickets for free. However, after a few rides, one of the kids feels nauseous and wants to go home.

Choice: How likely are you to stay at the amusement park for the day?

\section{Vignette 7: Tennis}

Adapted from Frisch (1993) Non-Harm/Care Conditions: Imagine that your child wants to learn to play tennis, so you (pay \$200 for them to take 12 tennis lessons) sign them up to take 12 free tennis lessons. After only two lessons, your child says that they are bored and do not want to play tennis anymore.

Harm/Care Conditions: Imagine that your child wants to learn to play tennis, so you (pay $\$ 200$ for them to take 12 tennis lessons) sign them up to take 12 free tennis lessons. 
After only two lessons, your child develops tennis elbow, and it is painful for them to continue to play tennis.

Choice: How likely are you to make your child finish the tennis lessons?

\section{Vignette 8: Pharmaceutical Company Executive}

Created by authors Non-Harm/Care Conditions: Imagine that you are the executive of a pharmaceutical company that spends \$1 billion to bring a new drug to market. You have invested (\$990 million) \$1 million into researching a new drug to treat high blood pressure. However, you find out that another pharmaceutical company begins marketing a similar drug, which is much more effective, cheaper to buy, and cheaper to produce than the drug your company is developing.

Harm/Care Conditions: Imagine that you are the executive of a pharmaceutical company that spends $\$ 1$ billion to bring a new drug to market. You have invested ( $\$ 990$ million) $\$ 1$ million into researching a new drug to treat high blood pressure. However, you learn that clinical trials of the drug find that it has many serious negative side effects that makes the drug dangerous to consume.

Choice: How likely are you to complete the project and bring the drug to market?

\section{Vignette 9: Letter *Removed from Experiment 3}

\section{Adapted from Strough et al. (2014) Non-Harm/Care} Conditions: Imagine that you are writing to your friend detailing a story that happened to you recently. You have written for (45 min) $5 \mathrm{~min}$ when you realize that if you had told the story another way it would have been funnier and easier to understand. It will take you about 10 more min to finish writing the letter.

Harm/Care Conditions: Imagine that you are writing a witness statement to the police about an assault that you witnessed recently. You have written for $(45 \mathrm{~min}) 5 \mathrm{~min}$ when you realize that if you had told the story another way it would be easier to understand and better help the victim win the case. It will take you about 10 more min to finish writing the statement.

Choice: How likely are you to continue writing?

\section{Vignette 10: Motor Vehicle Company Executive *Removed from Experiment 3}

Adapted from Olivola (2018) Non-Harm/Care Conditions: Imagine that you are the president of a motor vehicle company with a $\$ 100$ million research budget. You have invested (\$99 million) \$1 million dollars of the company's research budget across different unrelated projects. You have come up with a new design for a fuel-efficient car. Before production begins, you find out that another firm begins marketing a fuel-efficient car, which is much more fuel-efficient, economical and cheaper to produce than the car your company is building.

Harm/Care Conditions: Imagine that you are the president of a motor vehicle company with a $\$ 100$ million research budget. You have invested (\$99 million) \$1 million dollars of the company's research budget into across different unrelated projects. You have come up with a new design for a fuelefficient car. Before production begins, it is apparent that the prototype has flaws in the braking system that make the car dangerous to drive.

Choice: How likely are you to complete the project and bring the car to market?

\section{Vignette 11: Road Trip}

Adapted from Bruine de Bruin, Strough, and Parker (2014) Non-Harm/Care Conditions: Imagine that you and a friend have planned to go on a mini trip to spend time together at a destination $8 \mathrm{~h}$ away. Because your friend has no license, you are driving. The morning you intend to leave, the weather report says it will be raining all weekend. You think that it is "too bad" you already (drove halfway to the destination) planned and packed for the vacation, because the bad weather will affect the activities that you have planned for the trip.

Harm/Care Conditions: Imagine that you and a friend have planned to go on a mini trip to spend time together at a destination $8 \mathrm{~h}$ away. Because your friend has no license, you are driving. The morning you intend to leave, your friend feels sick. You think that it is "too bad" you already (drove halfway to the destination) planned and packed for the vacation, because your friend being sick will affect the activities that you have planned for the trip.

Choice: How likely are you to continue the trip?

Vignette 12: Medical Treatment *Removed from Experiment 3

Created by authors Non-Harm/Care Conditions: Imagine that a dependent family member has been receiving expensive medical treatment for acne for the past 2 months (which you have to pay for). You do not have to pay anything because it is fully covered by insurance. You got a prescription (paid $\$ 1,000$ ) for an 8-month supply of the medication. However, the treatment is only slightly working.

Low Sunk-Cost Moral dilemma: Imagine that a dependent family member has been receiving expensive medical treatment for acne for the past 2 months (which you have to pay for). You do not have to pay anything because it is fully covered by insurance. You got a prescription (paid \$1,000) for an 8-month supply of the medication. However, the treatment is causing painful joint inflammation.

Choice: How likely are you to continue with the current treatment? 


\section{References}

Arkes, H. R. (1996). The psychology of waste. Journal of Behavioral Decision Making, 9(3), 213-224. https://doi.org/10.1002/(SICI) 1099-0771(199609)9:3<213::AID-BDM230>3.0.CO;2-1

Arkes, H. R., \& Blumer, C. (1985). The psychology of sunk cost. Organizational Behavior and Human Decision Processes, 35(1), 124-140. https://doi.org/10.1016/0749-5978(85)90049-4

Armstrong, R. W., Williams, R. J., \& Barrett, J. D. (2004). The impact of banality, risky shift and escalating commitment on ethical decision making. Journal of Business Ethics, 53(4), 365-370. https://doi.org/ 10.1023/b:busi.0000043491.10007.9a

Brockner, J. (1992). The escalation of commitment to a failing course of action: Toward theoretical progress. The Academy of Management Review, 17(1), 39-61. https://doi.org/10.2307/258647

Bruine de Bruin, W., Strough, J., \& Parker, A. M. (2014). Getting older isn't all that bad: Better decisions and coping when facing "sunk costs". Psychology and Aging, 29(3), 642-647. https://doi.org/10. 1037/a0036308

Faul, F., Erdfelder, E., Lang, A., \& Buchner, A. (2007). G*Power 3: A flexible statistical power analysis program for the social, behavioral, and biomedical sciences. Behavior Research Methods, 39(2), 175191. https://doi.org/10.3758/bf03193146

Frisch, D. (1993). Reasons for framing effects. Organizational Behavior and Human Decision Processes, 54(3), 399-429. https://doi.org/10. 1006/obhd.1993.1017

Graham, J., Nosek, B. A., Haidt, J., Iyer, R., Koleva, S., \& Ditto, P. H. (2011). Mapping the moral domain. Journal of Personality and Social Psychology, 101(2), 366-385. https://doi.org/10.1037/ a0021847

Greenstein, M. J., \& Xu, X. (2015). My mug is valuable, but my partner's is even more so: Economic decisions for close others. Basic and Applied Social Psychology, 37(3), 174-187. https://doi.org/10. 1080/01973533.2015.1049347

Guler, I. (2007). Throwing good money after bad? Political and institutional influences on sequential decision making in the venture capital industry. Administrative Science Quarterly, 52(2), 248-285. https://doi.org/10.2189/asqu.52.2.248

Hackinger, J. (2019). Ignoring millions of euros: Transfer fees and sunk costs in professional football. Journal of Economic Psychology, 75, 102114. https://doi.org/10.1016/j.joep.2018.10.006

Haidt, J. (2008). Morality. Perspectives on Psychological Science, 3(1), 65-72. https://doi.org/10.1111/j.1745-6916.2008.00063.x

Haidt, J., Graham, J., \& Joseph, C. (2009). Above and below left-right: Ideological narratives and moral foundations. Psychological Inquiry, 20(2-3), 110-119. https://doi.org/10.1080/ 10478400903028573

Haidt, J., \& Joseph, C. (2004). Intuitive ethics: How innately prepared intuitions generate culturally variable virtues. Daedalus, 133(4), 5566. https://doi.org/10.1162/0011526042365555

Jensen, J. M., Conlon, D. E., Humphrey, S. E., \& Moon, H. (2011). The consequences of completion: How level of completion influences information concealment by decision makers. Journal of Applied Social Psychology, 41(2), 401-428. https://doi.org/10.1111/j.15591816.2010.00719.x

Kahneman, D., \& Tversky, A. (1979). Prospect theory: An analysis of decision under risk. Econometrica, 47, 263-291. https://doi.org/10. 2307/1914185

Larrick, R. P., Morgan, J. N., \& Nisbett, R. E. (1990). Teaching the use of cost-benefit reasoning in everyday life. Psychological Science, 1(6), 362-370. https://doi.org/10.1111/j.1467-9280.1990.tb00243.x

Lazar, S. (2018). Moral sunk costs. The Philosophical Quarterly, 68(273), 841-861. https://doi.org/10.1093/pq/pqy030

Lemhöfer, K., \& Broersma, M. (2012). Introducing LexTALE: A quick and valid lexical test for advanced learners of English. Behavior
Research Methods, 44(2), 325-343. https://doi.org/10.3758/ s13428-011-0146-0

Levin, I. P., Schneider, S. L., \& Gaeth, G. J. (1998). All frames are not created equal: A typology and critical analysis of framing effects. Organizational Behavior and Human Decision Processes, 76(2), 149-188. https://doi.org/10.1006/obhd.1998.2804

Loftus, G. R. (1978). On interpretation of interactions. Memory \& Cognition, 6(3), 312-319. https://doi.org/10.3758/BF03197461

Morsanyi, K., and Handley, S. J. (2008). How smart do you need to be to get it wrong? The role of cognitive capacity in the development of heuristic-based judgement. Journal of Experimental Child Psychology, 99(1), 18-36. https://doi.org/10.1016/j.jecp.2007.08. 003

Olivola, C. Y. (2018). The interpersonal sunk-cost effect. Psychological Science, 29(7), 1072-1083. https://doi.org/10.1177/ 0956797617752641

Rego, S., Arantes, J., \& Magalhães, P. (2018). Is there a sunk cost effect in committed relationships? Current Psychology, 37(3), 508-519. https://doi.org/10.1007/s12144-016-9529-9

Reynolds, S. J., \& Ceranic, T. L. (2007). The effects of moral judgment and moral identity on moral behavior: an empirical examination of the moral individual. Journal of Applied Psychology, 92(6), 1610 1624. https://doi.org/10.1037/0021-9010.92.6.1610

Roth, S., Robbert, T., \& Straus, L. (2015). On the sunk-cost effect in economic decision-making: a meta-analytic review. Business Research, 8(1), 99-138. https://doi.org/10.1007/s40685-014-00148

Rouder, J. N., Speckman, P. L., Sun, D., Morey, R. D., \& Iverson, G. (2009). Bayesian $t$ tests for accepting and rejecting the null hypothesis. Psychonomic Bulletin \& Review, 16(2), 225-237. https://doi. org/10.3758/PBR.16.2.225

Schönbrodt, F. D., Wagenmakers, E. J., Zehetleitner, M., \& Perugini, M. (2017). Sequential hypothesis testing with Bayes factors: Efficiently testing mean differences. Psychological Methods, 22(2), 322-339. https://doi.org/10.1037/met0000061

Schott, J. P., Scherer, L. D., \& Lambert, A. J. (2011). Casualties of war and sunk costs: Implications for attitude change and persuasion. Journal of Experimental Social Psychology, 47(6), 1134-1145. https://doi.org/10.1016/j.jesp.2011.06.002

Shanton, K., \& Goldman, A. (2010). Simulation theory. Wiley Interdisciplinary Reviews: Cognitive Science, 1(4), 527-538. https://doi.org/10.1002/wcs.33

Sherman, S. J., Cialdini, R. B., Schwartzman, D. F., \& Reynolds, K. D. (1985). Imagining can heighten or lower the perceived likelihood of contracting a disease: The mediating effect of ease of imagery. Personality and Social Psychology Bulletin, 11(1), 118-127. https://doi.org/10.1177/0146167285111011

Stewart, E. (2019). The Boeing 737 Max 8 crashes and controversy, explained. Retrieved from https://www.google.ca/amp/s/www.vox. com/platform/amp/2019/3/12/18262359/boeing-737-maxcontroversy-faa-trump

Street, M., \& Street, V. L. (2006). The effects of escalating commitment on ethical decision-making. Journal of Business Ethics, 64(4), 343 356. https://doi.org/10.1007/s10551-005-5836-z

Strough, J., Schlosnagle, L., Karns, T., Lemaster P., \& Pichayayothin, N. (2014). No time to waste: Restricting life-span temporal horizons decreases the sunk-cost fallacy. Journal of Behavioral Decision Making, 27(1), 78-94. https://doi.org/10.1002/bdm.1781

Team, R. C. (2019). A language and environment for statistical computing. Vienna, Austria: R Foundation for Statistical Computing; 2012. URL https://www.R-project.org.

Teger, A. I. (1980). Too much invested to quit. New York: Pergamon

Uzan, E. (2020). Moral sunk costs in war and self-defence. The Philosophical Quarterly https://doi.org/10.1093/pq/pqaa029

Wagenmakers, E. J., Krypotos, A. M., Criss, A. H., \& Iverson, G. (2012) On the interpretation of removable interactions: A survey of the field 
33 years after Loftus. Memory \& Cognition, 40(2), 145-160. https:// doi.org/10.3758/s13421-011-0158-0

Wald, A. (1945). Sequential tests of statistical hypotheses. The Annals of Mathematical Statistics, 16(2), 117-186. https://www.jstor.org/ stable/2235829

Whyte, G. (1986). Escalating commitment to a course of action: A reinterpretation. Academy of Management Review, 11(2), 311-321. https://doi.org/10.5465/amr.1986.4283111
Yglesias, M. (2019). The emerging 737 Max scandal, explained. Retrieved from https://www.vox.com/business-and-finance/2019/ 3/29/18281270/737-max-faa-scandal-explained

Publisher's note Springer Nature remains neutral with regard to jurisdictional claims in published maps and institutional affiliations. 